\title{
Does Fasting Modulate Physio-Psychological Responses to Emotional Pictures? An Analysis by MEG, VAS and Peripheral Physiological Markers
}

\author{
Kayo Mizoвe, ${ }^{*}$ Koichi Yokosawa,, \# Atsushi Shimojo, ${ }^{* *}$ Michitaka Ozaki ${ }^{*}$
}

\begin{abstract}
To relieve or manage stress, objective assessment of stress is required. Instead of direct assessment of stress, we have been investigating whether responses to emotional stimuli are modulated by stress. As emotional stimuli, 8 pleasant (P: high arousal and high emotional valence), 8 neutral (N: low arousal and moderate emotional valence), and 8 unpleasant (U: high arousal and low emotional valence) pictures were selected from the International Affective Picture System (Univ. of Florida) database and presented to 20 healthy volunteers for $3 \mathrm{~s}$ per picture. Psychological scores using a visual analog scale (VAS) indicate that the emotional valence of the selected pictures was perceived by the participants as expected. Magnetoencephalograms (MEG) and traditional physiological markers of heart rate and oxygen saturation of the peripheral artery $\left(\mathrm{SpO}_{2}\right)$ were recorded simultaneously. Amplitude modulations of alpha rhythm $(8-13 \mathrm{~Hz})$ by presentation of emotional pictures were compared in a normal (control) condition and in a fasting-induced stress condition. Data for 17 participants were analyzed by two-way [emotional category $(\mathrm{P} / \mathrm{N} / \mathrm{U}) \times$ condition (control/fasting)] repeated measures ANOVA and a multiple comparison test with Bonferroni's correction. Significant interaction in amplitude modulation was observed in the early stage (within $0.5-1.75 \mathrm{~s}$ from the start of picture presentation; $p=0.019$ ). In the control condition, alpha rhythms were more significantly suppressed by both pleasant and unpleasant pictures than by neutral pictures $(\mathrm{P}<\mathrm{N}: p=0.011, \mathrm{U}<\mathrm{N}: p=0.044)$. In the fasting condition, alpha rhythm was more significantly suppressed by unpleasant pictures than by pleasant pictures $(\mathrm{U}<\mathrm{P}: p=0.018)$. In other words, the amplitude depends on arousal in the control condition, while it depends on emotional valence in the fasting condition. Since it is known that alpha rhythm is suppressed by attention to visual input, these results suggest that attention to pleasant stimuli is lost under fasting-induced stress. On the other hand, heart rate and $\mathrm{SpO}_{2}$ were not different between the emotional categories and were not modulated by the fasting-induced stress. MEG seems to be practical and powerful for objective detection of prompt response to emotion and assessment of stress, probably because of its high temporal resolution and selectivity to a specific brain region.
\end{abstract}

Keywords: stress, emotion, magnetoencephalogram, fasting, biometry.

Adv Biomed Eng. 5: pp. 88-93, 2016.

\section{Introduction}

Excessive stress causes mental diseases. To relieve or manage stress, objective assessment of stress is required. Attempts to assess stress have been made by recording biochemical markers such as those in saliva and blood, or peripheral physiological markers such as blood pressure, heart rate, sweating, pupil size, and galvanic skin resistance [1].

Stress and emotions are closely related. In fact, the above-mentioned biochemical and physiological markers are also recorded to assess emotions. Cerebral electro-physiological methods including electroencephalography (EEG) and magnetoencephalography (MEG) have also been used to record brain responses to emotional stimuli [2]. It has been reported that sensory cortices respond promptly to emotional stimuli [3]. While conventional peripheral physiological markers, which are controlled

Received on January 27, 2016; accepted on April 14, 2016.

* Faculty of Health Sciences, Hokkaido University, Sapporo, Japan.

** Graduate School of Medicine, Hokkaido University, Sapporo, Japan.

\# N-12, W-5, Kita-Ku, Sapporo, Hokkaido 060-0812, Japan.

E-mail: yokosawa@med.hokudai.ac.jp by the autonomic nervous system, have limited temporal resolution, cerebral electro-physiological methods are useful for direct detection of prompt brain responses.

In this work, we investigated the interaction between stress and emotion [4]. MEG responses to emotional pictures under normal condition and fasting-induced stress condition were compared. Alpha rhythm $(8-13 \mathrm{~Hz})$, which is known to originate around the visual cortex and to reflect emotions and stress [5-7], was extracted by selecting appropriate sensors. Two traditional physiological markers; heart rate and oxygen saturation of the peripheral artery $\left(\mathrm{SpO}_{2}\right)$, were selected because of easy handling and compatibility to MEG recording. Heart rate, $\mathrm{SpO}_{2}$ and MEG were recorded simultaneously. However, to focus on the testing feasibility of MEG, the experimental setup was optimized to MEG recording. A visual analog scale (VAS) was also recorded as a psychological score.

The aim of this work was to determine the feasibility of MEG for objective assessment of emotion and stress by comparing with other psychological and conventional physiological methods.

\section{Methods}

The protocol of this study was approved by the Ethics Committee of the Faculty of Health Sciences and the Ethics Committee of the School of Medicine, Hokkaido University. Written informed con- 
sent was obtained from each participant prior to the experiments.

\subsection{Participants}

Twenty healthy volunteers (10 females, 10 males; age: mean \pm $\mathrm{SD}, 21.3 \pm 1.3$ years; range, $20-25$ years; all right-handed) participated in this study. The participants were randomly divided into two groups. Each group consisted of 5 males and 5 females. The participants in group 1 took part in the experiments under normal (control) condition first and then under fasting condition, and the participants in group 2 took part in the two experiments in the reverse order. For the fasting condition, each participant was instructed to fast from the night before the experiment for 12-15 hours. For the control condition, each participant spent their time as usual without specific instructions. The two experiments, under control and fasting conditions, were performed on different days for each participant.

\subsection{Materials}

From the International Affective Picture System (IAPS; University of Florida) [8], we selected pictures that have been validated for emotional content by more than 100 viewers: eight "pleasant" (abbreviated as P), eight "neutral" (N), and eight "unpleasant" (U) pictures. Each stimulus has emotional valence and arousal that were evaluated by viewers (Table 1). Pleasant $(\mathrm{P})$ pictures have high emotional valence and high arousal, neutral $(\mathrm{N})$ pictures have moderate emotional valence and low arousal, and unpleasant (U) pictures have low emotional valence and high arousal. The brightness of the selected pictures was standardized to mean

Table 1 Emotional pictures selected from the IAPS database.

\begin{tabular}{|c|c|c|c|c|}
\hline category & description & slide no. & valence & arousal \\
\hline & baby & 2071 & 7.86 & 5.00 \\
\hline pleasant & pregnant & 2155 & 6.78 & 5.43 \\
\hline \multirow[t]{2}{*}{ (P) } & family & 2156 & 7.12 & 4.34 \\
\hline & sunflower & 5001 & 7.16 & 3.79 \\
\hline average & garden & 5199 & 6.93 & 4.73 \\
\hline valence: 7.14 & field & 5725 & 7.09 & 3.55 \\
\hline \multirow[t]{3}{*}{ arousal: 4.78} & cupcakes & 7405 & 7.38 & 6.28 \\
\hline & french fries & 7460 & 6.81 & 5.12 \\
\hline & couple & 2390 & 5.40 & 3.57 \\
\hline neutral & girl & 2411 & 5.07 & 2.86 \\
\hline \multirow[t]{2}{*}{$(\mathbf{N})$} & outlet & 6150 & 5.08 & 3.22 \\
\hline & towel & 7002 & 4.97 & 3.16 \\
\hline average & spoon & 7004 & 5.04 & 2.00 \\
\hline valence: 5.09 & mug & 7009 & 4.93 & 3.01 \\
\hline \multirow[t]{3}{*}{ arousal: 3.08} & picnic table & 7026 & 5.38 & 2.63 \\
\hline & clock & 7211 & 4.81 & 4.20 \\
\hline & baby & 2053 & 2.47 & 5.25 \\
\hline unpleasant & black eye & 2345.1 & 2.26 & 5.50 \\
\hline \multirow[t]{2}{*}{ (U) } & stitches & 3185 & 2.81 & 5.48 \\
\hline & stitches & 3195 & 2.06 & 6.36 \\
\hline average & surgery & 3213 & 2.96 & 6.82 \\
\hline valence: 2.44 & dying man & 3230 & 2.02 & 5.41 \\
\hline \multirow[t]{2}{*}{ arousal: 5.74} & infant & 3350 & 1.88 & 5.72 \\
\hline & injecting & 9590 & 3.08 & 5.41 \\
\hline
\end{tabular}

brightness using appropriate image processing software.

The time sequence of a single epoch of the visual stimuli is shown in Fig. 1. The start cue, a cross-shaped fixation target at the center, was presented for $1 \mathrm{~s}$. After the start cue, one of the 24 emotional pictures was presented for $3 \mathrm{~s}$. Finally, a gray background was presented for $4-6 \mathrm{~s}$. The 24 pictures were presented in random order. Each picture was presented 10 times, and a total of 240 epochs were therefore presented in $40 \mathrm{~min}$. To maintain wakefulness of the participants, each experiment was divided into 3 sessions with approximately 1 -min breaks.

The visual stimuli were projected on a back projection screen located in a magnetically shielded room, which was essential for recording MEG signals, by a liquid-crystal projector located outside the shielded room. Illuminance in the shielded room was set at 12.0 Lux. Each participant watched the screen at a distance of $1 \mathrm{~m}$ in a sitting position. The visual angle of the horizontal side of each picture was 10 degrees in order to minimize eye movement.

\subsection{Measurements and Analysis \\ 2.3.1 Alpha rhythm}

MEG signals were recorded with a 76-channel helmet-shaped magnetometer system (customized; Elekta-Neuromag Oy, Helsinki, Finland) installed at Hokkaido University, Sapporo, Japan. The MEG signals were filtered with a passband of $0.03-200 \mathrm{~Hz}$ and were digitized at a sampling frequency of $600 \mathrm{~Hz}$.

In the analysis, the MEG signals of the three sessions were first concatenated. The concatenated signals were averaged over all epochs to obtain transient response to the start of picture presentation, and the signal sources were estimated as current dipoles [9]. The concatenated raw signals were filtered with a passband of $8-13 \mathrm{~Hz}$ and transformed to envelopes by Hilbert transform. The envelopes were averaged over epochs of each condition $(\mathrm{P} / \mathrm{N} / \mathrm{U})$. By the above process, envelopes (time courses of alpha rhythm amplitude) were obtained for all MEG sensors.

Assuming that alpha rhythm originates around the visual cortex, we selected ten sensors that were sensitive to the transient MEG responses evoked by the start of emotional picture presentation. The sensors were individually selected for each participant, but they were almost the same. Envelopes obtained by the ten sensors selected for each participant were averaged. The mean amplitudes within the following 4 time windows (tw) were then calculated: early (tw1: $0.50-1.75 \mathrm{~s}$ ) and late (tw2: 1.75-3.00 s) stages during presentation of the pictures, and early (tw3: 3.5$4.75 \mathrm{~s}$ ) and late (tw $4: 4.75-6.00 \mathrm{~s}$ ) stages after presentation of the pictures. Here, $0 \mathrm{~s}$ denotes the start of picture presentation. The

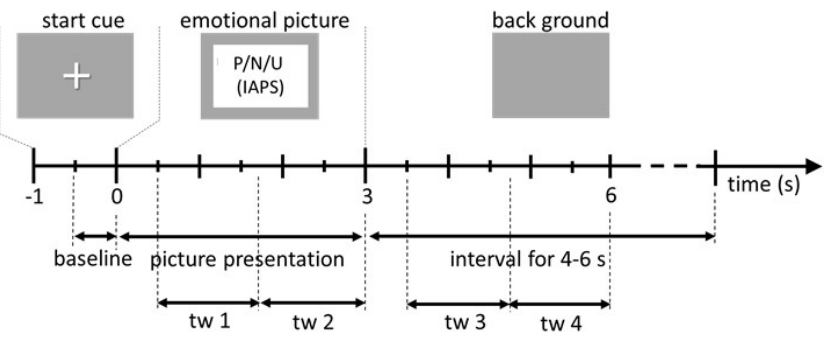

Fig. 1 Time sequence of a single epoch of emotional picture presentation. 
initial $0.5 \mathrm{~s}$ of presentation and the first $0.5 \mathrm{~s}$ after picture presentation were excluded because they include transient on- or off-responses. The mean amplitudes of the four time windows were normalized by the mean value during the baseline period ( -0.5 to $0 \mathrm{~s})$ to obtain relative modulation.

\subsubsection{Peripheral physiological markers}

Heart rate and $\mathrm{SpO}_{2}$ were also recorded during the experiments. The probe of an autonomic nerve activity monitoring device was fixed to the first toe of each participant. Due to the low temporal resolution and sluggishness of the heart rate and $\mathrm{SpO}_{2}$ values, their mean values during the presentation period $(0-3 \mathrm{~s})$ and the interval (3-7 s) were recorded by visual inspection.

\subsubsection{Psychological index}

Participants subjectively scored the emotional valence of each picture after each experiment. Each participant scored how he/ she felt about each picture by marking on a $100-\mathrm{mm}$ bar on a scoring sheet in which $0 \mathrm{~mm}$ denotes unpleasant and $100 \mathrm{~mm}$ denotes pleasant. The length between $0 \mathrm{~mm}$ and the marked position was measured (100-mm VAS).

\subsection{Statistical analysis}

Two-way repeated measures analysis of variance (ANOVA) was used for analysis with the following two within-subject factors: condition (control/fasting) and emotional category $(\mathrm{P} / \mathrm{N} / \mathrm{U})$. Post-hoc pairwise comparisons were conducted using Bonferroni's correction. The level of statistical significance was taken as $p<0.05$.

To clarify whether the differences between categories depend on "emotional valence" or "arousal", the multiple regression coefficient between alpha rhythm amplitude and emotional valence and that between alpha rhythm amplitude and arousal were calculated. The coefficients obtained were further analyzed by Wilcoxon's signed rank sum test at group level.

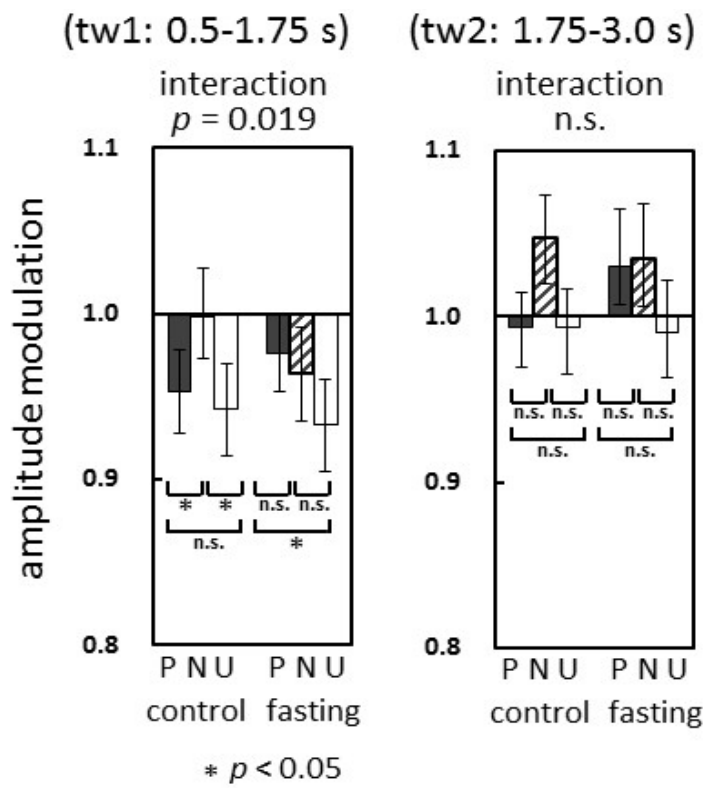

\section{Results}

Data for three participants were excluded from analysis because the signal source of the transient response could not be estimated in the visual cortex. Data for the remaining 17 participants $(9$ females, 8 males; mean \pm SD age, $21.4 \pm 1.3$ years; age range, 20 25 years) were analyzed statistically.

Figure 2 shows the time traces of alpha rhythm. The amplitudes were suppressed by the start cue and during the presentation of emotional pictures. After the pictures had disappeared, the amplitudes rebounded for $1-2 \mathrm{~s}$ and recovered. These results agree with the knowledge that alpha rhythm originating around the visual cortex is suppressed by visual input. Figure 3 shows the re-

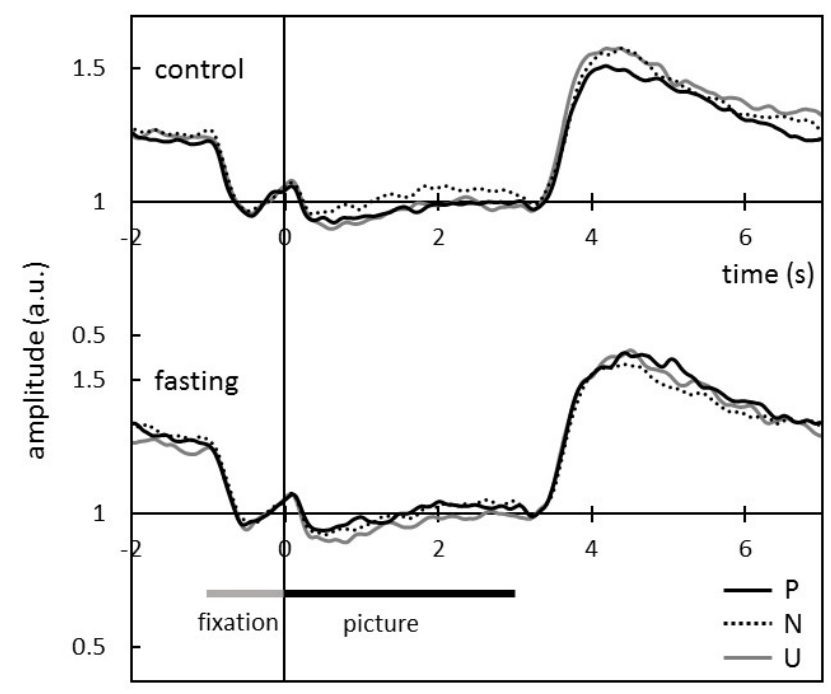

Fig. 2 Time traces of the amplitude of alpha rhythm. Amplitudes were normalized by the mean value in the baseline period $(-0.5-0 \mathrm{~s})$.

(tw3: 3.5-4.75 s) interaction

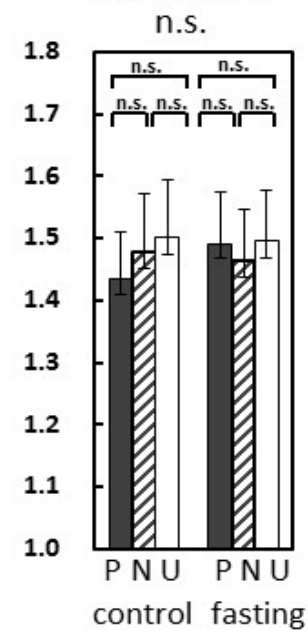

(tw4: 4.75-6.0 s) interaction

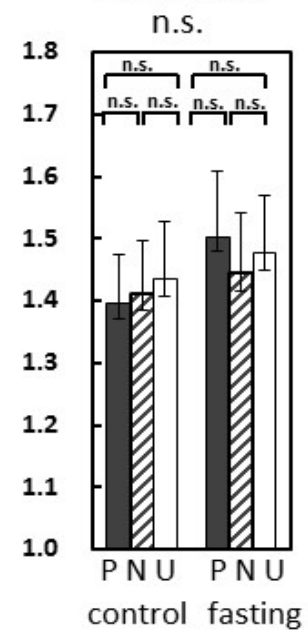

Fig. 3 Results of group level analysis of amplitude modulation of alpha rhythm in four time windows $(n=17) .0 \mathrm{~s}$ denotes the start of picture presentation. Error bar: standard error. 

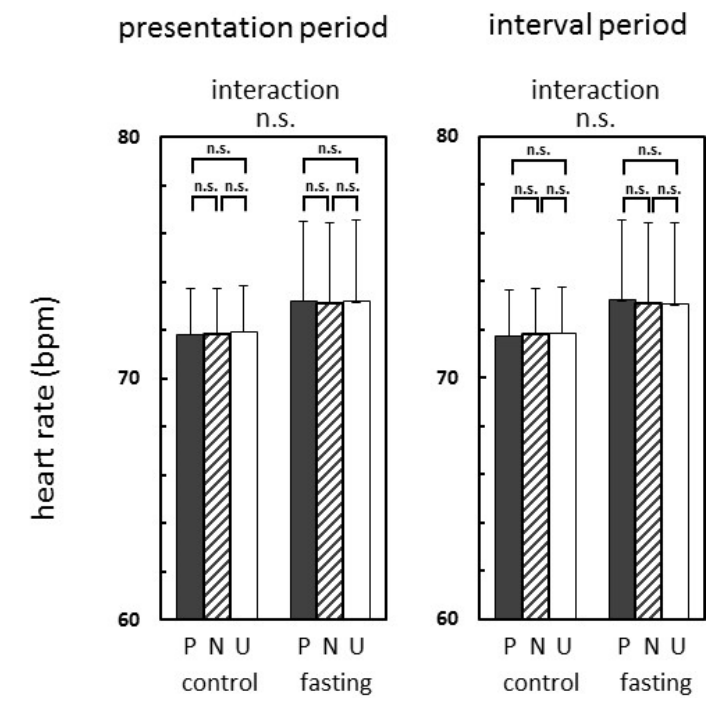

Fig. 4 Results of group level analysis of heart rate during and after picture presentation $(n=17)$. Error bar: standard error. bpm: beats per minute.
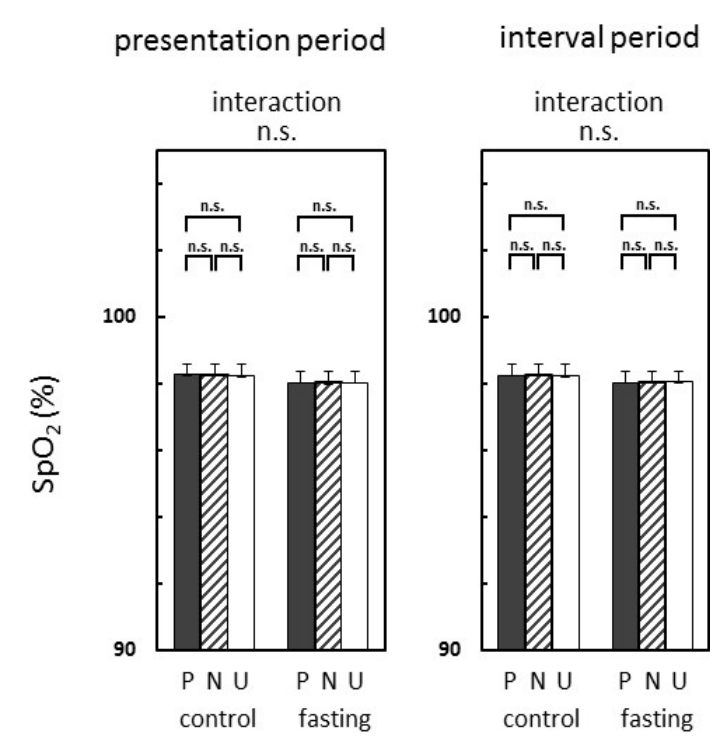

Fig. 5 Results of group level analysis of oxygen saturation of the peripheral artery $\left(\mathrm{SpO}_{2}\right)$ during and after picture presentation $(n=17)$. Error bar: standard error.

sults of statistical analysis of the amplitude modulation of alpha rhythm. A significant interaction between condition (control/fasting) and emotional category $(\mathrm{P} / \mathrm{N} / \mathrm{U})(p=0.019)$ and a main effect of emotional category $(p=0.010)$ were observed in the early stage of picture presentation ( $t w 1: 0.50-1.75 \mathrm{~s}$ ). In the control condition, the amplitudes of $\mathrm{P}$ and $\mathrm{U}$ were significantly suppressed compared with that of $\mathrm{N}(\mathrm{P}<\mathrm{N}: p=0.011, \mathrm{U}<\mathrm{N}: p=$ 0.044), while the amplitude modulation between $\mathrm{P}$ and $\mathrm{U}$ was not significant. On the other hand, in the fasting condition, the amplitude of $\mathrm{U}$ was significantly suppressed compared with that of $\mathrm{P}$ ( $\mathrm{U}<\mathrm{P}: p=0.018)$. No interaction or main effect was observed in the other time windows; tw2, tw3, and tw4.

There were no significant interactions or main effects for heart rate and $\mathrm{SpO}_{2}$ (Figs. 4 and 5). No interaction in VAS score

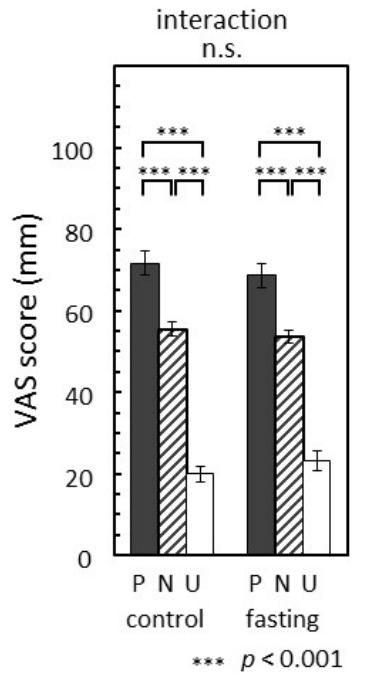

Fig. 6 Results of group level analysis of 100-mm VAS $(n=17)$ of emotional pictures. Error bar: standard error.

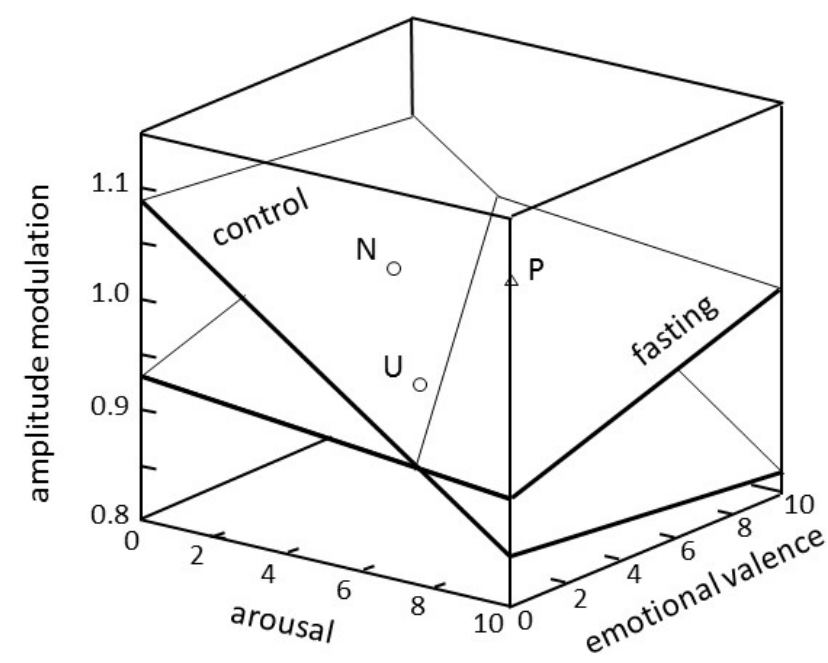

Fig. 7 Three-dimensional map showing tendency of "alpha rhythm amplitude modulation" against "emotional valence" or "arousal". Two planes of the control and fasting conditions are constructed through three points of $\mathrm{P}, \mathrm{N}, \mathrm{U}$ for each condition. Only $\mathrm{N}$ and $\mathrm{U}$ in the control condition and $\mathrm{P}$ in the fasting condition are visible.

was observed between condition (control/fasting) and emotional category $(\mathrm{P} / \mathrm{N} / \mathrm{U})$. The emotional category showed a main effect $(p<0.001)$, and significant differences were found between control and fasting conditions (Fig. 6).

The group factor (group 1: control condition followed by fasting condition, group 2: fasting condition followed by control condition) was also analyzed statistically. No main effect was found, indicating that there was no order effect.

To clarify the results for alpha rhythm, a three-dimensional map between "emotional valence", "arousal", and "alpha rhythm amplitude modulation" is shown in Fig. 7. Amplitude modulation is derived from the data in Fig. 3 (tw1), while emotional valence and arousal of $\mathrm{P} / \mathrm{N} / \mathrm{U}$ are from those in Table 1. Two planes of control and fasting conditions are constructed through three 
points of $\mathrm{P}, \mathrm{N}, \mathrm{U}$ for each condition. For the control condition, amplitude declines along the arousal axis but is flat along the valence axis; while for the fasting condition, amplitude increases along the valence axis but is unchanged along the arousal axis. To confirm these tendencies statistically, the multiple regression coefficient between amplitude and emotional valence and that between amplitude and arousal were calculated for each participant. Since the coefficients obtained were not normally distributed, Wilcoxon's signed rank sum test was performed. The multiple regression coefficient between amplitude modulation and arousal is negative and significant $(p=0.009)$ in the control condition, indicating that higher arousal suppresses alpha rhythm to a greater extent. In contrast, the coefficient between amplitude modulation and emotional valence is positive and significant $(p=0.011)$ in the fasting condition, meaning that lower valence (i.e., more unpleasant) suppresses alpha rhythm to a greater extent.

\section{Discussion}

The results for the 100-mm VAS indicate that emotional valence of the selected pictures perceived by the subjects was similar to that in the IAPS technical report [8]. Heart rate and $\mathrm{SpO}_{2}$ were not different between emotional categories. However, alpha rhythm, which originates from the primary visual cortex, is suppressed differently by presentation of emotional pictures, and the degree of suppression was significantly different between emotional categories. Moreover, fasting-induced stress modulated the difference. The results of multiple regression analysis demonstrated that higher arousal causes greater alpha rhythm suppression in the control (normal) condition, while lower (negative) emotional valence causes greater alpha rhythm suppression in the fasting condition.

It is known that alpha rhythm is suppressed by attention to visual input [10]. In the control condition, looking at emotional (P and $\mathrm{U}$ ) pictures with high arousal suppressed alpha rhythm to similar extent. This result suggests that pictures with high arousal receive more attention than do neutral pictures with low arousal.

In the fasting condition, the unpleasant pictures and pleasant pictures modulated alpha rhythm differently, indicating that alpha rhythm suppression depends on emotional valence. The results shown in Fig. 3 suggest that this difference was due to little suppression by pleasant pictures. A possible interpretation is that attention to pleasant stimuli is lost under stressful condition, while attention to unpleasant stimuli remains. Unpleasant stimuli are sometimes warning signs, and attention to unpleasant stimuli may therefore be essential for survival even under stressful conditions. The loss of attention to pleasant stimuli under stress may be in line with emotional disorder caused by depression [11].

Since the response time of traditional peripheral physiological markers is limited by the autonomic nervous system, too short duration of picture presentation may account for why the markers are not different between emotional categories and not modulated by stress. Besides, alpha rhythm is modulated even in the early stage of picture presentation. Nevertheless, while the experimental setup was optimized to MEG, high temporal resolution of MEG seems to be practical and powerful for detecting prompt response to emotion and its modulation under a stressful condition.

In line with previous finding, the present study confirms that attention causes alpha rhythm suppression. However, there is still room to distinguish between the effects of attention and emotion. A stressful condition should also be distinguished from physical conditions such as possible hypoglycemia caused by fasting. Other stressors should be investigated in a future study.

\section{Conclusion}

Recording of alpha rhythm by MEG suggested that both pleasant and unpleasant visual stimuli receive attention under normal conditions, while attention to pleasant stimuli is lost under a fasting-induced stressful condition. MEG, a cerebral electro-physiological tool that can probe cerebral response directly may be a useful tool for objective assessment of emotion and stress.

\section{Conflict of interest}

We have no conflicts of interest relationship with any companies or commercial organizations based on the definition of the Japanese Society of Medical and Biological Engineering.

\section{Acknowledgement}

This work was supported by JSPS Grant-in-Aid for Scientific Research 25670940.

\section{References}

1. Brown DE: Measuring hormonal variation in the sympathetic nervous system. In: Gillian HI, Gary DJ eds, Measuring stress in humans: A practical guide for the field. Cambridge University Press, New York, pp. 94-121, 2007.

2. Junghöfer M, Peyk P, Flaisch T, Schupp HT: Neuroimaging methods in affective neuroscience: selected methodological issues. Prog Brain Res. 156, pp. 123-143, 2006.

3. Thönnessen H, Boers F, Dammers J, Chen Y-H, Norra C, Mathiak $\mathrm{K}$ : Early sensory encoding of affective prosody: neuromagnetic tomography of emotional category changes. NeuroImage. 50, pp. 250-259, 2010.

4. Mizobe K, Yokosawa K, Sawabe Y, Ota S, Kawamura Y, Hangai S, Shimojo A, Ozaki M: An impact of "fasting" upon bio-responses to emotional pictures: An analysis by MEG, VAS and biological markers (pulse and $\mathrm{SpO}_{2}$ ). J Jpn Biomagn and Bioelectromagn Soc. 27(1), pp. 160-161, 2014. (in Japanese)

5. Onoda K, Okamoto Y, Shishida K, Hashizume A, Ueda K, Yamashita H, Yamawaki S: Anticipation of affective images and event-related desynchronization (ERD) of alpha activity: An MEG study. Brain Res. 1151, pp. 134-141, 2007.

6. Balconi M, Brambilla E, Falbo L: Appetitive vs. defensive responses to emotional cues: Autonomic measures and brain oscillation modulation. Brain Res. 1296, pp. 72-84, 2009.

7. Nishifuji S, Sato M, Maino D, Tanaka S: Effect of acoustic environment on EEG during mental task. J Life Support Eng. 22(3), pp. 10-18, 2010. (in Japanese)

8. Lang PJ, Bradley MM, Cuthbert BN: International affective picture system (IAPS): Technical manual and affective ratings. Technical report A-8. University of Florida, Gainesville, Florida, 2008.

9. Salmelin R: Multi-dipole modeling in MEG. In: Hansen PC, Kringrlbach, and Salmelin R eds, MEG: An introduction to methods. Oxford University Press, New York, pp. 124-155, 2010.

10. Klimesch W: Alpha-band oscillations, attention, and controlled access to stored information. Trends Cogn Sci. 16(12), pp. 606617, 2012.

11. Hyman SE, Cohen JD: Disorders of mood and anxiety. In: Kandel ER, Schwartz JH, Jessell TM, Siegelbaum SA, Hudspeth AJ 
eds, Principles of Neural Science, 5th ed. McGrawhill, New York, pp. 1402-1424, 2000.

\section{Kayo Mizoвe}

Kayo Mrzobe was born in Obihiro, Japan, in 1968. She graduated from Department of Nursing, College of Medical Technology, Hokkaido University in 1990 and has worked as a licensed nurse for Hokkaido University Hospital. She transferred to Hokkaido University in 2000 and studying critical

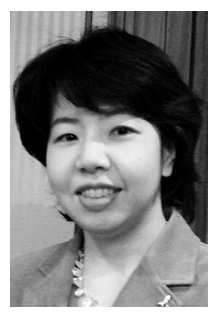
care nursing and stress management during perioperative period as a lecturer from 2005. She received B.S., M.S. and Ph.D. degrees in Graduate School of Business Administration from Hokkai Gakuen University in 2000, 2002, 2010.

\section{Koichi YoKosawa}

Koichi Yokosawa was born in Sendai, Japan, in 1962. He received B.S. and M.S. degrees in physics from the Faculty of Science, Hokkaido University in 1984 and 1986 respectively, and a Ph.D. degree in systems and information engineering from the Faculty of Engineering, Hokkaido Uni-

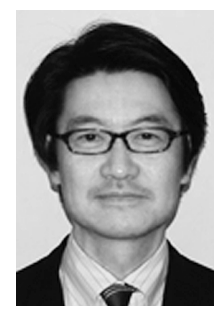
versity in 1998. He joined the Central Research Laboratory, Hitachi, Ltd. in 1986, and was engaged in medical instrument research and development. He transferred to Hokkaido University in 2007 where he holds a professorship and is now engaged mainly in studying brain function using magnetoencephalography.

\section{Atsushi Sнimojo}

Atsushi Sнiмоло was born in Fukushima, Japan, in 1975. He received M.S. degree from Graduate School of Information Science and Technology, Hokkaido University in 2010. Now, he is studying brain function using magnetoencephalography, in the Graduate School of Medicine, Hokkaido University.

\section{Michitaka OzaKI}

Michitaka OzAKI was born in Kurashiki, Japan, in 1959. He graduated from Okayama University School of Medicine (M.D., 1984) and acquired Ph.D. in Okayama University Faculty of Medicine (1988). His clinical career started as a surgeon and has been involved in gastroenterological and trans-

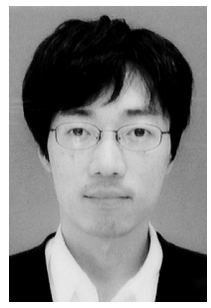
plantation surgery. Research-wise, his major interest has been biological stress, especially intracellular oxidative stress and cellular response. In Hokkaido University, he newly started the study to monitor organ functions/conditions non-invasively and chronologically by developing optic probes. 